\title{
The clinical value of hematological neutrophil and monocyte parameters in the diagnosis and identification of sepsis
}

\author{
Wenping Zhang ${ }^{1}$, Zhongming Zhang ${ }^{1}$, Shiyao Pan ${ }^{2}$, Jin $\mathrm{Li}^{2}$, Yanmei Yang ${ }^{1}$, Huan $\mathrm{Qi}^{2}$, Jiabin Xie ${ }^{1}$, \\ Jiuxin $Q u^{1}$
}

${ }^{1}$ Department of Clinical Laboratory, The Third People's Hospital of Shenzhen, Southern University of Science and Technology, National Clinical Research Center for Infectious Diseases, Shenzhen, China; ${ }^{2}$ Hematology Application and Research Department, Shenzhen Mindray Bio-Medical Electronic Co., Ltd., Shenzhen, China

Contributions: (I) Conception and design: W Zhang; (II) Administrative support: J Qu, H Qi; (III) Provision of study materials or patients: Z Zhang, Y Yang; (IV) Collection and assembly of data: J Xie; (V) Data analysis and interpretation: S Pan, J Li; (VI) Manuscript writing: All authors; (VII) Final approval of manuscript: All authors.

Correspondence to: Jiuxin Qu. Department of Clinical Laboratory, The Third People's Hospital of Shenzhen, Southern University of Science and Technology, National Clinical Research Center for Infectious Diseases, 29th Bulan Road, Longgang District, Shenzhen 518112, China. Email: qujiuxin@163.com.

Background: Sepsis is a life-threatening condition of organ dysfunction caused by the host's disordered immune response to infection. It has a high fatality rate and seriously endangers human health. Rapid and accurate treatment plays an important role in the reduction of septic mortality. This study aimed to investigate the clinical value of hematological parameters neutrophil (NEU)-X, NEU-Y, monocyte (MON)-X, and MON-Y in sepsis, and compare their values with that of with C-reactive protein (CRP).

Methods: We collected dipotassium ethylenediaminetetraacetic acid (EDTA-K2) anticoagulant blood samples from a total of 267 patients with positive bacterial culture and 260 healthy physical check-up patients. Participants were divided into three groups: a normal control group $(n=260)$, bacterial infection group ( $\mathrm{n}=196)$, and a sepsis group ( $\mathrm{n}=71)$.

Results: Median values of NEU-X, NEU-Y, MON-X, MON-Y, and CRP in the sepsis group were significantly higher than those in the control group and the bacterial infection group $(\mathrm{P}<0.0001)$. The area under the receiver operating characteristic curve (AUC) of NEU-X, NEU-Y, MON-X, MON-Y, and CRP for the diagnosis of sepsis was 0.751 (sensitivity 76.1\%, specificity 58.2\%), 0.877 (87.3\%, 72.1\%), 0.791 (77.6\%, 65.9\%), 0.695 (71.6\%, 51.4\%), and 0.790 (72.5\%, 70.2\%), respectively. In addition, blood smear examination results showed that NEU-X value was positively correlated with the degree of toxic granulation in neutrophils.

Conclusions: The parameters NEU-X, NEU-Y, and MON-X can be used as indicators for the differential diagnosis of sepsis with comparable diagnostic efficacy to CRP. Compared to CRP, these hematological parameters are easier to obtain, more convenient, and have economic benefits.

Keywords: Hematological parameters; C-reactive protein (CRP); sepsis; toxic granulation; morphological changes

Submitted Sep 22, 2021. Accepted for publication Nov 16, 2021.

doi: 10.21037/atm-21-5639

View this article at: https://dx.doi.org/10.21037/atm-21-5639

\section{Introduction}

Sepsis is a life-threatening condition of organ malfunction caused by a disordered host immune response to infection. Every year, more than 19 million people experience sepsis and 6 million die. With a fatality rate of more than 25, sepsis seriously endangers human health (1-3). It is a progressive disease, which gradually advances from local infection to septic shock, and the mortality increases with the course 
of the disease $(4,5)$. Many articles have reported that early interventional therapy can effectively improve the survival rate of patients with sepsis (6). Kumar reported that if sepsis patents were not treated within $72 \mathrm{~h}$ of onset, their survival rates would drop by about $7.7 \%$ per hour (7). However, diagnosis of sepsis is difficult due to its lack of obvious and specific signs or symptoms. At present, blood culture is considered the most reliable technique for diagnosis of sepsis, but it is a time-consuming procedure, and falsenegative cases are common (8). Although other infection indicators, such as C-reactive protein (CRP), procalcitonin (PCT), and interleukin-6 (IL-6) are widely used in the diagnosis of sepsis, they also have disadvantages, such as insufficient sensitivity and specificity, being affected by certain diseases, and the associated additional examination fees increasing the economic burden of patients. Therefore, how to distinguish infection types and diagnose sepsis early, conveniently, and economically has become an urgent problem to be solved in clinical laboratories.

When sepsis occurs, peripheral blood neutrophils are stimulated and activated by pathogens, resulting in a series of morphological changes such as the appearance of toxic granulation, vacuolization, left shift, and chromatin density increase. Monocytes are also activated, resulting in increased cell volume and nucleic acid content. It has been reported that the more severely infected patients are, the more significant these morphological changes are $(9,10)$. Mathy and Tejeswini also suggested that observing the content of neutrophil toxic granulation and immature granulocytes in peripheral blood smear can provide reference information for the diagnosis of bacterial infection $(9,11)$.

The Mindray BC-6800 Plus automated hematology analyzer (Mindray, Shenzhen, China) uses SF-Cube detection technology to obtain the leukocytes volume, cellular complexity, nucleic acid content, and other characteristic information, and automatically outputs quantitative detection parameters that can describe the cell morphology, such as NEU-X, NEU-Y, MON-X and MON-Y (NEU represents neutrophils; MON stands for monocytes; $\mathrm{X}$ indicates the center of gravity position of the sideward scatter light signal and represents the cellular complexity; $\mathrm{Y}$ indicates the center of gravity position of sideward fluorescence laser signal, and represents the content of nucleic acid in cells). All these parameters can vividly reflect the morphological changes of peripheral blood cells.

This study aimed to investigate the potential clinical value of NEU-X, NEU-Y, MON-X, and MON-Y, using the receiver-operating characteristic (ROC) curve to determine the best diagnostic threshold in the diagnosis of sepsis, and compare their clinical performances to CRP. In addition, microscopic blood smear examination was performed to confirm whether hematological parameters can truly reflect the morphological change of leukocytes. We present the following article in accordance with the STARD reporting checklist (available at https://dx.doi.org/10.21037/atm-215639).

\section{Methods}

\section{Patients}

A total of 527 dipotassium ethylenediaminetetraacetic acid (EDTA-K2) anticoagulant whole blood samples were collected from patients admitted to the emergency department, internal medicine department, infection department, intensive care unit (ICU) and Physical Examination Department of the Third People's Hospital of Shenzhen from December 2018 to June 2019. All tests were completed within $6 \mathrm{~h}$ after sample collection.

We retrospectively collected three groups for this study. Group 1 consisted of normal healthy physical check-up individuals without any signs or symptoms of infection or inflammation ( $\mathrm{n}=260)$, including 132 males and 128 females, aged 19-72 years with an average age of 35.6 years (SD $=11.8$ ). Group 2 consisted of patients with positive culture result who were diagnosed with bacterial infection $(n=196)$, including 151 males and 45 females, aged 20-94 years with an average age of 55.5 years $(\mathrm{SD}=17.9)$. Group 3 consisted of sepsis patients with positive culture results $(n=71)$, including 49 males and 22 females, aged 20-89 years old with an average age of 50.8 years $(\mathrm{SD}=17.7)$.

The criteria for enrollment of sepsis patients were based on the Third International Consensus Definitions for Sepsis and Septic Shock (Sepsis-3) published in 2016 (12). The consensus recommends a diagnosis of sepsis with a suspected infection and a Sequential Organ Failure Assessment (SOFA) score of 2 or greater. Samples with clinical diagnosis of bacterial infection and SOFA score less than 2 were enrolled into the bacterial infection group.

All procedures performed in this study involving human participants were in accordance with the Declaration of Helsinki (as revised in 2013). This study was approved by the ethics committee of The Third People's Hospital of Shenzhen (No. 2019-118). Due to the study's retrospective 
Table 1 Kruskal-Wallis test results for control, bacterial infection, and sepsis groups

\begin{tabular}{lcccc}
\hline Groups & 01 control $(\mathrm{n}=260)$ & 02 bacterial infection $(\mathrm{n}=196)$ & 03 sepsis $(\mathrm{n}=71)$ & $P$ value \\
\hline NEU-X & $333.60(316.70,354.66)^{\mathrm{a}}$ & $357.10(335.75,383.50)$ & $383.20(350.77,421.97)^{\mathrm{b}}$ & $<0.0001$ \\
NEU-Y & $433.50(412.51,453.96)$ & $447.00(423.00,471.20)^{\mathrm{c}}$ & $514.30(472.88,556.07)^{\mathrm{a}}$ & $<0.0001$ \\
MON-X & $197.70(190.40,209.26)$ & $202.00(193.32,210.88)^{\mathrm{d}}$ & $217.70(206.83,234.20)^{\mathrm{a}}$ & $<0.0001$ \\
MON-Y & $993.00(944.68,1,046.11)$ & $960.00(903.92,1,015.00)^{\mathrm{a}}$ & $1,033.60(974.02,1,125.97)^{\mathrm{e}}$ & $<0.0001$ \\
CRP & $1.630(0.933,2.577)$ & $57.090(33.897,107.155)^{\mathrm{a}}$ & $124.350(68.667,212.820)^{\mathrm{a}}$ & $<0.0001$ \\
\hline
\end{tabular}

${ }^{a}$, indicates statistically significant differences with other groups $(P<0.0001) ;{ }^{b}$, indicates a statistically significant difference with the bacterial infection group $(P<0.01) ;{ }^{c}$, indicates a statistically significant difference with the control group $(P<0.01)$; ${ }^{d}$, indicates statistically different from the control group $(P<0.05)$; , represents a statistically significant difference with the control group $(P<0.01)$. NEU, neutrophils; $M O N$, monocytes; $X$ indicates the center of gravity position of the sideward scatter light signal and represents the cellular complexity; $\mathrm{Y}$ indicates the center of gravity position of sideward fluorescence laser signal, and represents the content of nucleic acid in cells; CRP, C-reactive protein.

nature, the requirement to obtain signed informed consent from the patients was waived.

\section{Instruments}

We measured NEU-X, NEU-Y, MON-X, and MON-Y with a Mindray BC-6800 Plus automatic hematology analyzer. The CRP was measured by ADVIA 2400 automatic biochemical-immune analyzer (Siemens, Munich, Germany).

\section{Data collection}

The EDTA-K2 anticoagulant whole blood samples and serum samples were collected from 527 participants. The whole blood samples were analyzed by Mindray BC6800 Plus automatic hematology analyzer according to the laboratory operating procedures, and the parameters of neutrophils and monocytes were recorded. The serum samples were measured using the ADVIA 2400 automatic biochemical immune analyzer, and the CRP results were recorded. In addition, in order to preliminarily confirm whether NEU-X can truly reflect the degree of toxic granulation within neutrophils, 5 samples with different NEU-X levels were selected for microscopic blood smear examination.

\section{Statistical analysis}

The Kolmogorov-Smirnov test was used to test the normal distribution of continuous variables. Data with a normal distribution were expressed as the mean and SD and comparisons between three groups were made using analysis of variance (ANOVA) tests, and Turkey-Kramer multiple comparisons test were used to test the significance between two groups. Data with non-normal distribution were expressed as the median and interquartile range (IQR value) and comparisons between three groups were made using the Kruskal-Wallis test, and Dunn's multiple comparisons test were used to test the significance between two groups. A 2 -sided $\mathrm{P}$ value $<0.05$ was considered statistically significant.

Statistical analyses were performed with the software SPSS 21.0 (IBM Corp., Armonk, NY, USA), and GraphPad Prism 8.0 (GraphPad Software, San Diego, CA, USA) was used to plot graphs.

\section{Results}

\section{Differences in parameters among three participant groups}

Using 260 results from the control group, 196 results from the bacterial infection group, and 71 results from the sepsis group, Kruskal-Wallis tests were performed to compare the three groups. The results for NEU-X, NEU-Y, MON-X, MON-Y, and CRP are shown in Table 1 and Figure 1A-1E. As shown in Table 1, the median values of NEU-X, NEU-Y, MON-X, MON-Y, and CRP in the sepsis group were significantly higher than those in the normal control group and the bacterial infection group $(\mathrm{P}<0.0001)$. The median values of NEU-X, NEU-Y, MON-X, and CRP in the bacterial infection group were higher than those in the normal control group $(\mathrm{P}<0.05)$. As shown in Figure $1 E$, although the median CRP level was high in the bacterial infection group, it was significantly higher in the sepsis group, with a significant difference $(\mathrm{P}<0.0001)$. 


\section{Clinical value in diagnosis of sepsis}

We performed ROC analysis, and the area under the ROC curve (AUC) was calculated to evaluate the performances of these parameters in sepsis diagnosis. The optimal cutoff values were determined based on Youden's index (sensitivity + specificity - 1), then the diagnostic sensitivity and specificity were calculated. As shown in Figure $1 F$, NEU-X, NEU-Y, MON-X, MON-Y, and CRP had good diagnostic performances in the diagnosis of sepsis. The AUCs of the 4 parameters were $0.751,0.877,0.791$, and 0.695 respectively, while the AUC of CRP was 0.790 . When the cutoff values of NEU-X, NEU-Y, MON-X, and MON-Y were 350.3, $457.8,206.5$, and 981.8 , the sensitivities were $74.6 \%$, $87.3 \%, 77.6 \%$, and $71.6 \%$, and the specificities were $58.2 \%$, $72.1 \%, 65.9 \%$, and $51.4 \%$, respectively. When the cutoff value of CRP was 75.43 , the sensitivity and specificity were $75.43 \%$ and $72.5 \%$ (Table 2). The AUC of NEU-Y was larger than CRP, suggesting that it had a better diagnostic efficacy (Figure 1F). The diagnostic value of MON-X, NEU-X, and CRP were shown to be considerable; however, the ROC curve area of MON-Y for sepsis diagnosis was smaller than CRP, suggesting that the diagnostic efficacy was slightly weaker than CRP.

\section{Blood smear examination}

In order to preliminarily confirm whether NEU-X can truly reflect the toxic granulation of neutrophils, 5 levels of NEU-X samples with the values of 296.9, 351.2, 401.5, 447.3 , and 504.5 were selected for microscopic blood smear examination. The images of neutrophil toxic granulation under the microscope are shown in Table 3. The results showed that when the NEU-X value was 296.9, the cytoplasmic granules of neutrophils decreased. When the NEU-X value was 351.2, the morphology of neutrophils was generally normal, and neutrophil specific granules were present in the cytoplasm, but no toxic granulation was found. When the NEU-X value was 401.5, the cytoplasmic granules increased significantly, and the cytoplasmic color become darker due to the increase of granules, but no obvious toxic granules were observed. When the value of NEU-X was 447.3, the cytoplasm was filled with toxic granulation, which became darker in color. At the same time, some neutrophils showed nuclear shift to the left, and band form neutrophil increased significantly. When the NEU-X value reached 504.5, the amount of toxic granulation increased further, and the cytoplasmic color was darker than other levels. There was a significant positive correlation between NEU-X value and the number of toxic granulation neutrophils. In other words, NEU-X could reflect the number and thickness of toxic granulation in the cytoplasm of neutrophils.

\section{Discussion}

The early diagnosis of sepsis has a significant guiding role in the treatment and prognosis of the disease; however, the current auxiliary indicators used in the laboratory, such as microbial culture, CRP, PCT, and IL-6, have certain disadvantages, which cannot satisfactorily meet the needs of clinical diagnosis. Therefore, finding an effective, accurate, and low-cost method for sepsis detection has always been an important challenge for clinical laboratories.

Microscopic blood smear examination is a traditional method for detection of leukocyte morphological changes, but this method is time-consuming and laborious, requires laboratory technicians to have high morphological experience, and the results are highly subjective. Therefore, microscopic blood smear examination has not been widely applied in clinical laboratories to date.

The Mindray BC-6800 Plus automatic hematology analyzer can automatically quantitatively measure and output the cellular complexity and nucleic acid content of neutrophils and monocytes, and express the levels as NEU-X, NEE-Y, MON-X, and MON-Y. The detection speed of this method is much faster than the traditional microscopic blood smear examination method, the test results are more objective, and the labor cost is significantly less.

Several studies have shown that these morphological parameters have a favorable role in the diagnosis of sepsis. Crouser and Polilli et al. proposed that when the body is invaded by a pathogen, monocytes are activated to resist the pathogen, resulting in an acute increase in monocyte size (13-15). The monocyte morphological parameter monocyte distribution width (MDW) can reflect the size of monocytes and then be used in the early diagnosis of patients with sepsis. Their experimental results showed that MDW has a higher diagnostic performance than white cell count (WBC) in the diagnosis of sepsis. Ayres, Cornet, and Dinsdale also found that changes in the cellular complexity and nucleic acid content of neutrophils can reflect the infection status of the body, assisting the early diagnosis of sepsis (16-21).

It is well known that when the body is invaded by 

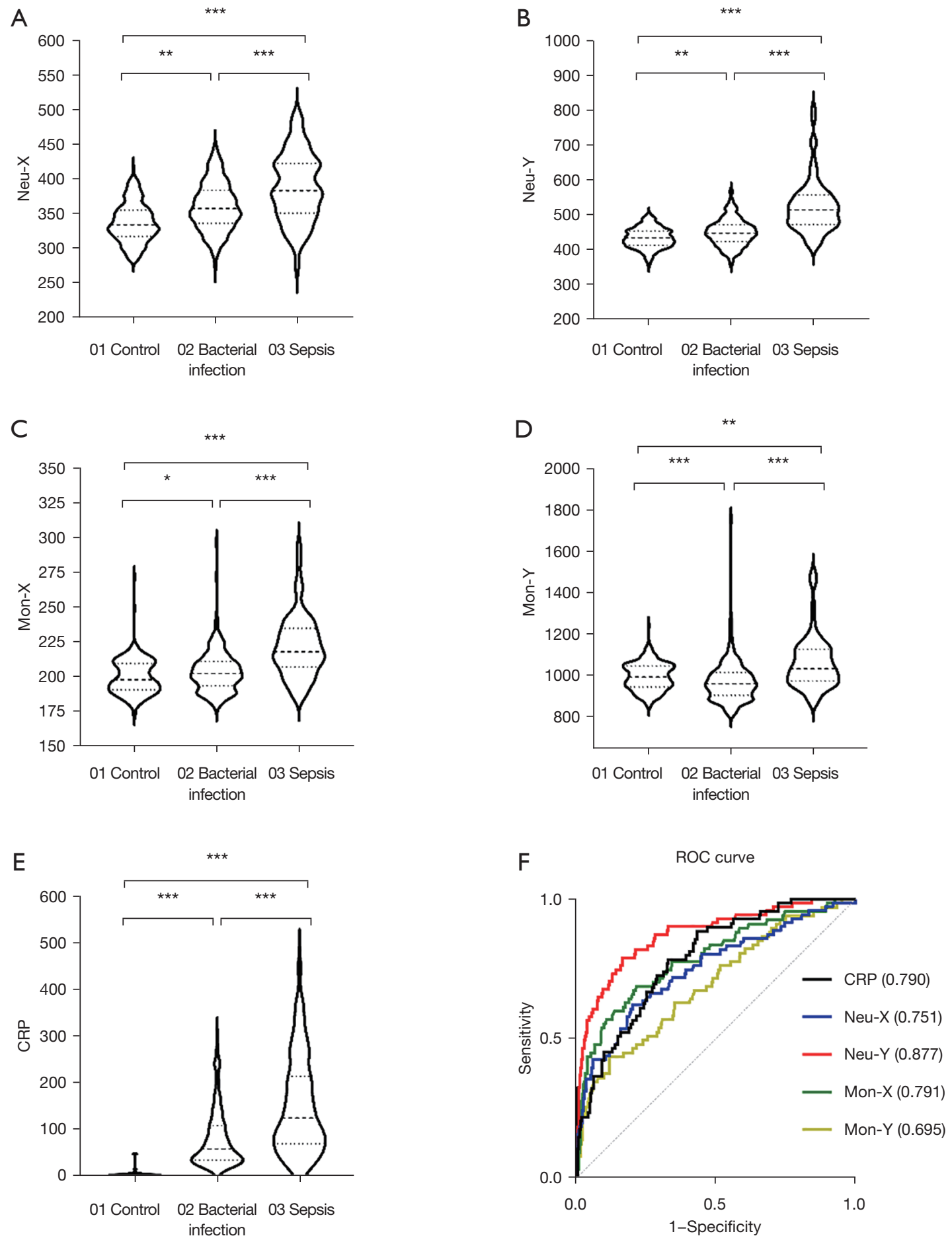

Figure 1 Violin plots and ROC curve for comparisons of hematological parameters and CRP. (A-E) Violin plots (25th and 75th percentiles, median) for comparisons of NEU-X, NEU-Y, MON-X, and MON-Y with CRP among the control, bacterial infection and sepsis groups. ***, indicates a significant difference at $\mathrm{P}<0.0001$; **, indicates $0.0001<\mathrm{P}<0.001$; * indicates $0.01<\mathrm{P}<0.05$. (F) $\mathrm{ROC}$ curve comparing the values of NEU-X, NEU-Y, MON-X, and MON-Y with CRP in the diagnosis of sepsis. NEU, neutrophils; MON, monocytes; X indicates the center of gravity position of the sideward scatter light signal and represents the cellular complexity; $\mathrm{Y}$ indicates the center of gravity position of sideward fluorescence laser signal, and represents the content of nucleic acid in cells; CRP, C-reactive protein; ROC, receiver operating characteristic. 
Table 2 ROC analysis results for the four parameters and CRP

\begin{tabular}{|c|c|c|c|c|c|}
\hline Parameters & AUC & $95 \% \mathrm{Cl}$ & Cut-off & Sensitivity (\%) & Specificity (\%) \\
\hline NEU-Y & 0.877 & $0.825-0.921$ & 457.8 & 87.3 & 72.1 \\
\hline MON-X & 0.791 & $0.720-0.849$ & 206.5 & 77.6 & 65.9 \\
\hline MON-Y & 0.695 & $0.616-0.758$ & 981.9 & 71.6 & 51.4 \\
\hline
\end{tabular}

NEU, neutrophils; MON, monocytes; $X$ indicates the center of gravity position of the sideward scatter light signal and represents the cellular complexity; $\mathrm{Y}$ indicates the center of gravity position of sideward fluorescence laser signal, and represents the content of nucleic acid in cells; CRP, C-reactive protein; ROC, receiver operating characteristic; AUC, area under ROC curve; 95\% Cl, 95\% confidential interval.

bacteria, neutrophils and monocytes are activated to perform bactericidal and phagocytic functions to resist pathogenic microorganisms. Cytokines and proinflammatory cytokines [such as granulocyte-macrophage colony-stimulating factor (GM-CSF) and IL-6] are activated and released when cytokine storm occurs in patients with sepsis. These cytokines can stimulate and activate neutrophils, monocytes, and lymphocytes, and enhance cellular immune function. After neutrophils are activated, a series of morphological changes will occur, including the appearance of toxic granulation, vacuolization, and Dohle bodies, the left shift, and the increase of nucleic acid substances (12). When monocytes are activated to phagocytize foreign pathogens, they also cause changes in cellular complexity and nucleic acid content $(22,23)$. These changes in cell morphology can be detected by hematology analyzer. The morphological changes of these white blood cells can more intuitively reflect the response of the body when bacteria invade, and may strengthen the accuracy of sepsis diagnosis.

The results of this study showed that neutrophil and monocyte parameters NEU-X, NEU-Y, MON-X, and MON-Y were significantly increased in the sepsis group. There were significant differences between the control group and bacterial infection group. This suggests that NEU-X, NEU-Y, MON-X, and MON-Y can be used for diagnosis of sepsis, and can distinguish sepsis from bacterial infection.

In addition, ROC curve analysis showed that when NEU-Y was used in the diagnosis of sepsis, the AUC of 0.877 was higher than that of CRP (0.790), and the sensitivity and specificity were $87.3 \%$ and $72.1 \%$. This indicates that NEU-Y has a good screening predictive performance as a sepsis screening project, and its diagnostic performance is better than that of CRP. The AUCs of NEU-X and MON-X were 0.751 and 0.791 respectively, which were similar to CRP, although with slightly better sensitivity and slightly lower specificity. However, the AUC value of MON-Y was 0.695 , and the sensitivity specificity were $71.6 \%$ and $51.4 \%$, respectively, suggesting that MON-Y is less effective in the diagnosis of sepsis.

It is worth mentioning that CRP and PCT may be affected in patients with liver or kidney failure. Since CRP originates from the liver, impaired function of the liver in patients may lead to dysfunction of CRP synthesis, which cannot reflect the infection of the body correctly. The PCT level is significantly increased in patients with chronic kidney disease, but this increase may be unrelated to sepsis (24-26). In this case, NEU-X, NEU-Y, and MON-X appear to be more effective. Their readings are not influenced by primary diseases such as liver and kidney diseases. In this case, NEU-X, NEU-Y, and MON-X are more useful in bacterial infection, especially sepsis.

This study also examined 5 different levels of NEU-X blood smear, and the results showed that NEU-X can correctly reflect the cellular complexity of the neutrophils: the greater the NEU-X value, the higher the complexity, the more likely to have the presence of toxic granulation. There was a significant positive correlation between NEU-X value and the number of toxic granulation neutrophils.

The result of NEU-X, NEU-Y, MON-X, and MON-Y are generated along with the complete blood count (CBC) on the Mindray BC-6800 Plus so that the measurements are rapid, low cost, and convenient. Clinicians can quickly identify sepsis patients based on the results of NEU-X, NEU-Y, MON-X, and MON-Y, and the patient's clinical symptoms. After patients with sepsis are identified, clinical 
Table 3 Images of neutrophil blood smear with different NEU-X levels

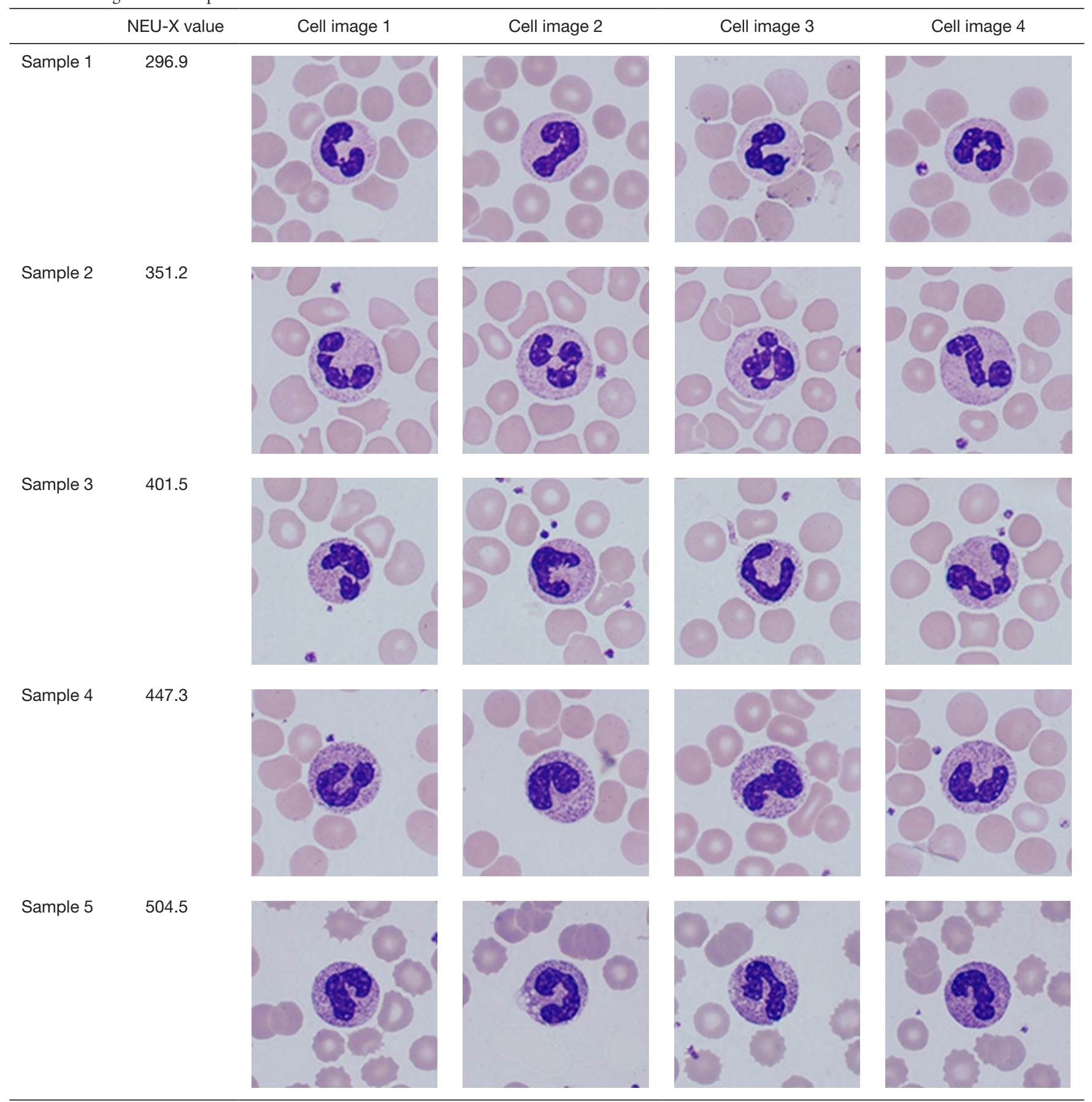

Mindray SC-120 Automatic Slide Maker is used to make standard blood smears. Wright-Giemsa is used as the staining method. Sample 1 shows the reduction of neutrophil cytoplasmic granules; in sample 2, the morphology of neutrophils was generally normal and no toxic granulation was found; in sample 3, neutrophil cytoplasmic granules increased more than normal, and granules filled the whole cytoplasm; in sample 4, there was obvious toxic granulation in the cytoplasm and band form neutrophils significantly increased; in sample 5, a large volume of toxic granulation was found throughout the cytoplasm. With the increase in the quantity of toxic granulation, the cytoplasm became darker with the appearance of vacuoles. NEU, neutrophils; $X$ indicates the center of gravity position of the sideward scatter light signal and represents the cellular complexity. 


\section{Page 8 of 9}

doctors can establish reasonable anti-infective treatment plans. The survival rate of patients will improve, and the abuse of antibiotics can be avoided.

In conclusion, NEU-X, NEU-Y, and MON-X are useful parameters for the diagnosis of sepsis, and display superior convenience and performance to that of CRP.

\section{Acknowledgments}

Funding: This work was supported by Guangdong Basic and Applied Basic Research Foundation (2020A1515010586), Science and Technology Program of Shenzhen (JCYJ20190809144005609), the Special Support Fund of Shenzhen for Introduced High-Level Medical Team (SZSM201412005).

\section{Footnote}

Reporting Checklist: The authors have completed the STARD reporting checklist. Available at https://dx.doi. org/10.21037/atm-21-5639

Data Sharing Statement: Available at https://dx.doi. org/10.21037/atm-21-5639

Conflicts of Interest: All authors have completed the ICMJE uniform disclosure form (available at https://dx.doi. org/10.21037/atm-21-5639). SP, JL and HQ are from Shenzhen Mindray Bio-Medical Electronic Co., Ltd., Shenzhen, China. The other authors have no conflicts of interest to declare.

Ethical Statement: The authors are accountable for all aspects of the work in ensuring that questions related to the accuracy or integrity of any part of the work are appropriately investigated and resolved. All procedures performed in this study involving human participants were in accordance with the Declaration of Helsinki (as revised in 2013). This study was approved by the ethics committee of The Third People's Hospital of Shenzhen (No. 2019-118). Due to the study's retrospective nature, the requirement to obtain signed informed consent from the patients was waived.

Open Access Statement: This is an Open Access article distributed in accordance with the Creative Commons Attribution-NonCommercial-NoDerivs 4.0 International License (CC BY-NC-ND 4.0), which permits the non-

\section{Zhang et al. The hematological parameters help diagnosis sepsis}

commercial replication and distribution of the article with the strict proviso that no changes or edits are made and the original work is properly cited (including links to both the formal publication through the relevant DOI and the license). See: https://creativecommons.org/licenses/by-nc-nd/4.0/.

\section{References}

1. Perner A, Cecconi M, Cronhjort M, et al. Expert statement for the management of hypovolemia in sepsis. Intensive Care Med 2018;44:791-8.

2. Prescott HC, Angus DC. Postsepsis Morbidity. JAMA 2018;319:91.

3. Reinhart K, Daniels R, Kissoon N, et al. Recognizing Sepsis as a Global Health Priority - A WHO Resolution. N Engl J Med 2017;377:414-7.

4. Bone RC, Balk RA, Cerra FB, et al. Definitions for sepsis and organ failure and guidelines for the use of innovative therapies in sepsis. The ACCP/SCCM Consensus Conference Committee. American College of Chest Physicians/Society of Critical Care Medicine. Chest 1992;101:1644-55.

5. Cheng B, Xie G, Yao S, et al. Epidemiology of severe sepsis in critically ill surgical patients in ten university hospitals in China. Crit Care Med 2007;35:2538-46.

6. Angus DC, Linde-Zwirble WT, Lidicker J, et al. Epidemiology of severe sepsis in the United States: analysis of incidence, outcome, and associated costs of care. Crit Care Med 2001;29:1303-10.

7. Kumar A, Roberts D, Wood KE, et al. Duration of hypotension before initiation of effective antimicrobial therapy is the critical determinant of survival in human septic shock. Crit Care Med 2006;34:1589-96.

8. Gerdes JS. Diagnosis and management of bacterial infections in the neonate. Pediatr Clin North Am 2004;51:939-59, viii-ix.

9. Tejeswini V, Kande S, Premalatha P, et al. Correlation of granularity index with toxic granulation of neutrophils by manual microscopy and C-reactive protein neutrophils by manual microscopy and C-reactive protein. Journal of Dental and Medical Sciences 2012;3:35-9.

10. Al-Gwaiz LA, Babay HH. The diagnostic value of absolute neutrophil count, band count and morphologic changes of neutrophils in predicting bacterial infections. Med Princ Pract 2007;16:344-7.

11. Mathy KA, Koepke JA. The clinical usefulness of segmented vs. stab neutrophil criteria for differential leukocyte counts. Am J Clin Pathol 1974;61:947-58. 
12. Singer M, Deutschman CS, Seymour CW, et al. The Third International Consensus Definitions for Sepsis and Septic Shock (Sepsis-3). JAMA 2016;315:801-10.

13. Crouser ED, Parrillo JE, Seymour C, et al. Improved Early Detection of Sepsis in the ED With a Novel Monocyte Distribution Width Biomarker. Chest 2017;152:518-26.

14. Crouser ED, Parrillo JE, Seymour CW, et al. Monocyte Distribution Width: A Novel Indicator of Sepsis-2 and Sepsis-3 in High-Risk Emergency Department Patients. Crit Care Med 2019;47:1018-25.

15. Polilli E, Sozio F, Frattari A, et al. Comparison of Monocyte Distribution Width (MDW) and Procalcitonin for early recognition of sepsis. PLoS One 2020;15:e0227300.

16. Ayres LS, Sgnaolin V, Munhoz TP. Immature granulocytes index as early marker of sepsis. Int J Lab Hematol 2019;41:392-6.

17. Cornet E, Boubaya M, Troussard X. Contribution of the new XN-1000 parameters NEUT-RI and NEUT-WY for managing patients with immature granulocytes. Int J Lab Hematol 2015;37:e123-6.

18. Dinsdale RJ, Devi A, Hampson P, et al. Changes in novel haematological parameters following thermal injury: A prospective observational cohort study. Sci Rep 2017;7:3211.

19. Mammen J, Choudhuri J, Paul J, et al. Cytomorphometric Neutrophil and Monocyte Markers May Strengthen the

Cite this article as: Zhang W, Zhang Z, Pan S, Li J, Yang Y, Qi H, Xie J, Qu J. The clinical value of hematological neutrophil and monocyte parameters in the diagnosis and identification of sepsis. Ann Transl Med 2021;9(22):1680. doi: 10.21037/atm-21-5639
Diagnosis of Sepsis. J Intensive Care Med 2018;33:656-62.

20. Buoro S, Manenti B, Seghezzi M, et al. Innovative haematological parameters for early diagnosis of sepsis in adult patients admitted in intensive care unit. J Clin Pathol 2018;71:330-5.

21. Urrechaga E, Bóveda O, Aguirre U. Role of leucocytes cell population data in the early detection of sepsis. J Clin Pathol 2018;71:259-66.

22. Henderson RB, Hobbs JA, Mathies M, et al. Rapid recruitment of inflammatory monocytes is independent of neutrophil migration. Blood 2003;102:328-35.

23. Italiani P, Boraschi D. From Monocytes to M1/M2 Macrophages: Phenotypical vs. Functional Differentiation. Front Immunol 2014;5:514.

24. Wu SC, Liang CX, Zhang YL, et al. Elevated serum procalcitonin level in patients with chronic kidney disease without infection: A case-control study. J Clin Lab Anal 2020;34:e23065.

25. Zheng W, Liang X, Shui L, et al. Serum Procalcitonin Correlates with Renal Function in Hepatitis B VirusRelated Acute-on-Chronic Liver Failure. Cell Physiol Biochem 2018;50:1794-803.

26. Jeeha R, Skinner DL, De Vasconcellos K, et al. Serum procalcitonin levels predict acute kidney injury in critically ill patients. Nephrology (Carlton) 2018;23:1090-5.

(English Language Editor: J. Jones) 